\title{
Validação da Escala de Autoconceito e Autoestima para Pré-adolescentes (EAAPA) e Análise da Estrutura Organizativa do Autoconceito
}

\author{
Validation of the Self-concept and Self-esteem Scale for Pre-adolescents (EAAPA) \\ and Analysis of Structural Organization of Self-concept
}

\author{
Francisco Peixoto ${ }^{1}$, Lourdes Mata ${ }^{1}$, Vera Monteiro ${ }^{1}$, Cristina Sanches ${ }^{1}$, Rui Bártolo-Ribeiro ${ }^{1}$ e \\ Joana Pipa ${ }^{1}$
}

\begin{abstract}
Resumo
Este estudo teve como objetivo a análise das propriedades psicométricas de uma escala de autoconceito e autoestima e da estrutura organizativa do autoconceito em pré-adolescentes. Participaram 774 estudantes Portugueses do $5^{\circ}$ e $6^{\circ}$ anos de escolaridade, que responderam a uma escala que avalia sete dimensões específicas do autoconceito (competência escolar, competência atlética, aceitação social, aparência física, competência a língua materna, competência a matemática, comportamento) e a autoestima. As análises fatoriais confirmatórias demonstraram a multidimensionalidade do autoconceito sendo o modelo bi-fatorial com dois fatores globais (autoconceito académico e não académico) aquele que apresentou um melhor ajustamento aos dados. Este modelo tem a vantagem de integrar características estruturais do autoconceito que em estudos anteriores foram consideradas incompatíveis.
\end{abstract}

Palavras-chave: Escala de Autoconceito e Autoestima para Pré-adolescentes (EAAPA), autoconceito, autoestima, psicometría, pré-adolescentes

\begin{abstract}
The main goal of this research was the analysis of the psychometric proprieties of a self-concept and selfesteem scale and the study of the self-concept organizational structure in pre-adolescents. Participants were 774 Portuguese students attending 5th and 6th grades. The scale used assesses seven specific dimensions of self-concept (school competence, athletic competence, social acceptance, physical appearance, native language competence, mathematics competence, behavior), as well as self-esteem. Confirmatory factor analyses supported the multidimensionality of self-concept, and a bi-factor model with two global factors (academic self-concept and non-academic self-concept) was the one that presented a better fit. This model enables to integrate structural characteristics of self-concept that seemed to be incompatible in previous research.
\end{abstract}

Keywords: Self-concept and Self-esteem Scale for Pre-adolescents (EAAPA), self-concept, self-esteem, psychometrics, pre-adolescents

Este trabalho integra uma investigação mais alargada financiada pela Fundação para a Ciência e Tecnologia (PTDC/CPE-CED/121358/2010).

${ }^{1}$ ISPA-Instituto Universitário - Centro Investigação Educação. Lisboa, Portugal.

E-mail para contacto: fpeixoto@ispa.pt 


\section{Introdução}

O autoconceito é muitas vezes considerado como importante mediador na obtenção de um adequado desempenho nos múltiplos contextos onde a pessoa se insere (Jackson, Thomas, \& Marsh, 2001; Marsh, Craven, \& Martin, 2006; Peixoto, 2003) e pode ser definido como o conjunto de crenças que cada um possui acerca de si próprio (Hattie, 1992; Peixoto, 2003), formadas a partir das interpretações das experiências vividas no meio que as envolve (Marsh, 1993).

No domínio social e escolar, o desenvolvimento do autoconceito é reconhecido como um objetivo a alcançar, constituindo-se igualmente como uma variável mediadora de outros constructos. Um autoconceito positivo associa-se a variáveis como bem-estar psicológico, motivação, rendimento académico e escolhas vocacionais (Barros, 2012; GómezMaqueo, Eguiarte, \& Patiño, 2007; Peixoto, 2011; Peixoto \& Almeida, 2010; Peixoto \& Carvalho, 2009). Neste sentido, a promoção do autoconceito torna-se essencial para o bem-estar e sucesso académico dos sujeitos, sendo de igual forma importante a existência de instrumentos válidos que o permitam caracterizar.

Um marco importante na investigação sobre o autoconceito foi a publicação do artigo de Shavelson, Hubner e Stanton (1976), no qual propunham um modelo teórico que reconhecia a sua organização multidimensional e hierárquica e que se tornaria cada vez mais diferenciada com o aumento da idade. Mais especificamente, este modelo considerava a existência de um autoconceito global no topo da hierarquia e de dimensões mais específicas na base da estrutura. A um nível intermédio, Shavelson et al. (1976) consideravam a existência de um autoconceito académico e de um autoconceito não académico, sendo que o autoconceito académico integraria as dimensões mais específicas relacionadas com as disciplinas escolares, (e.g. autoconceito matemático e o autoconceito a língua materna). Quanto ao autoconceito não académico este organizar-se-ia ainda em mais três dimensões intermédias, os autoconceitos social, emocional e físico, subdividindo-se estes em autoconceitos específicos (e.g., relação com os pares, aparência física e competência atlética).

Um dos princípios enunciados por Shavelson et al. (1976) seria o de que o autoconceito se tornaria mais diferenciado com a idade. Esta ideia é partilhada por autores como Byrne (1996), Harter (2012) e Hattie (1992) que referem que, efetivamente, os estudantes pré-adolescentes avaliam as suas capacidades de forma cada vez mais diferenciada, justificando-se a apresentação de uma estrutura multidimensional para esta faixa etária.

Neste sentido, alguns trabalhos têm sido desenvolvidos para a caracterização e compreensão da estrutura do autoconceito em crianças e pré-adolescentes (e.g. Marsh, Barnes, Cairns, \& Tidman, 1984; Raimundi, López, Cataldi, \& Bugallo, 2011). Num estudo baseado no modelo teórico de Shavelson et al. (1976), Marsh et al. (1984) desenvolveram o SDQ que lhes permitiu, de forma empírica, suportar e posteriormente rever o modelo teórico subjacente. Avaliaram a estrutura fatorial do autoconceito de crianças dos 6 aos 11 anos, a frequentar entre o $2^{\circ}$ e o $5^{\circ}$ ano. Os seus resultados evidenciaram um aumento da diferenciação da estrutura multidimensional do autoconceito com o avançar da idade, principalmente no que diz respeito às dimensões académicas. Nesta amostra, foi possível observar uma estrutura multidimensional do autoconceito comportando sete fatores (competência física, aparência física, relação com os pares, relação com os pais, verbal/língua materna, matemática e escolar), obtendo-se correlações mais fracas entre os fatores nos alunos mais velhos, o que evidencia uma maior especificidade e individualidade de cada um destes fatores com o incremento da idade.

Um outro estudo conduzido por Molina, Raimundi, López, Cataldi e Bugallo (2011), junto de uma amostra de estudantes de Buenos Aires com idades compreendidas entre os 8 e 13 anos e utilizando a adaptação da escala Self Perception Profile for Children, demonstrou uma boa qualidade de discriminação dos itens, levando a crer que a escala mede constructos relativamente independentes. Os seus resultados corroboram a ideia de que o autoconceito é um constructo multifacetado, tornando-se as suas facetas mais 
diferenciadas à medida que os sujeitos avançam na escolaridade (Molina et al., 2011; Peixoto \& Almeida, 2011).

Ainda que sejam variados os estudos que apoiam a estrutura multidimensional do autoconceito, que agrega várias facetas da vida dos sujeitos (e.g., Cheng \& Watkins, 2000; Hattie, 1992; Marsh \& Shavelson, 1985; Marsh, 1990; Molina et al., 2011; Shavelson \& Bolus, 1982), a sua estrutura hierárquica parece menos clara e mais complexa do que o previamente proposto por Shavelson e colaboradores (1976; Marsh \& Hocevar, 1985; Marsh \& Shavelson, 1985).

Neste sentido, os estudos de Marsh e Hocevar (1985) e de Marsh e Shavelson (1985) encontraram alguma sustentação para a estrutura multidimensional proposta por Shavelson et al. (1976), porém os seus resultados evidenciaram uma estrutura hierárquica mais complexa do que a previamente proposta, com as dimensões verbal e matemática pouco correlacionadas. Estes resultados sugeriam que a organização das dimensões de ordem superior seria mais complexa, levando Marsh e colaboradores a proporem um modelo hierárquico que diferenciava os autoconceitos verbal e matemático, como dimensões de ordem superior: o modelo de Marsh/Shavelson (Marsh, 1990; Marsh \& Ayotte, 2003; Marsh \& Hocevar, 1985; Marsh \& Shavelson, 1985).

Marsh e Hocevar (1985) conduziram análises fatoriais confirmatórias à estrutura do autoconceito de alunos do $2^{\circ}$ ao $5^{\circ}$ ano de escolaridade, testando o modelo de Shavelson et al. (1976). Através da análise de vários modelos hierárquicos, os resultados revelaram um melhor ajustamento do modelo que considerava uma dimensão não académica e duas dimensões académicas de ordem superior: verbal/língua materna e matemática, comparativamente aos modelos que consideravam apenas um fator global de ordem superior e aos que contemplavam dois fatores de ordem superior: académico e não académico.

Com o intuito de explicar a fraca correlação entre as dimensões do autoconceito académico, Marsh (1986; Marsh \& Hau, 2004) desenvolveu o quadro de referência interno/externo (I/E). De acordo com este modelo, os autoconceitos matemático e verbal são ambos influenciados tanto por comparações internas como externas. A ideia subjacente é a de que os estudantes desenvolvem a sua perceção de competência académica baseada em dois tipos de comparação: interna, onde o aluno compara a sua competência numa dada disciplina com a sua competência em outras disciplinas; e externa, através da qual o sujeito compara a sua competência em determinadas disciplinas com as competências dos seus colegas nessas mesmas disciplinas (Marsh, 1986; Marsh \& Hau, 2004).

Contrariamente, Abu-Hilal e Bahri (2000), no seu estudo com 569 alunos árabes do $5^{\circ}, 6^{\circ}, 8^{\circ}$ e $9^{\circ}$ anos de escolaridade, observaram correlações elevadas entre as dimensões do SDQ. Para além disso, obtiveram valores de correlação elevados entre o autoconceito verbal e o autoconceito matemático nos alunos mais novos ( $r=.83)$ e uma correlação, apesar de substancialmente mais fraca, ainda assim significativa, para os alunos mais velhos $(\mathrm{r}=.37)$. Os resultados encontrados neste estudo poderão evidenciar um efeito cultural sobre o autoconceito, demonstrando que este poderá não se organizar da mesma forma em culturas distintas (Abu-Hilal \& Bahri, 2000; Cheng \& Watkins, 2000).

Comparativamente aos alunos do ensino secundário, estudos no âmbito da avaliação da estrutura do autoconceito em alunos mais novos são mais escassos, especificamente com alunos do $5^{\circ}$ e $6^{\circ}$ ano, embora algumas investigações corroborem a ideia da existência de uma estrutura multidimensional nestas faixas etárias (e.g., Faria $\&$ Fontaine, 1990).

Apesar da relativa escassez de estudos sobre a organização do autoconceito em pré-adolescentes, alguns estudos existem, nomeadamente a partir da adaptação de instrumentos de avaliação do autoconceito, mas nos quais é possível discernir alguns dos aspetos associados à organização deste constructo. Também em Portugal foram, ao longo do tempo, surgindo adaptações para a população portuguesa de instrumentos que analisassem e caracterizassem o autoconceito. Faria e Fontaine (1990), na adaptação portuguesa do SDQ-I, junto de estudantes do $5^{\circ}, 7^{\circ}$ e $9^{\circ}$ anos, recorrendo a análises fatoriais exploratórias, comprovaram a estrutura multidimensional de oito dimensões 
subjacente à escala utilizada. No entanto, apesar de relativamente bem definida, a solução fatorial obtida evidenciou a existência de alguns itens a saturar em dimensões não completamente congruentes com o seu conteúdo (e.g. autoconceito escolar e autoconceito verbal, autoconceito global e autoconceito escolar).

Os estudos sobre a estrutura hierárquica do autoconceito incidindo sobre $\mathrm{o}$ autoconceito académico, apesar de maioritários, não se esgotam nesta faceta de ordem superior do autoconceito. Deste modo, é possível encontrar estudos que pretenderam averiguar a existência de uma estrutura hierárquica noutras áreas como o autoconceito social (Byrne \& Shavelson, 1996; Leung, Marsh, Yeung, \& Abduljabbar, 2015), o autoconceito físico (Marsh, Hey, Johnson, \& Perry, 1997) e o autoconceito artístico (Vispoel, 1995).

As investigações aqui apresentadas sugerem que o sujeito estabelece avaliações das suas competências em diferentes domínios da sua vida e que estas se organizam hierarquicamente. No que se refere à investigação sobre a organização do autoconceito académico, o modelo de Marsh/Shavelson tem sido largamente predominante. No entanto, recentemente Brunner e colaboradores (Brunner et al., 2010; Brunner, Keller, Hornung, Reichert, \& Martin, 2009) propuseram um outro modelo de organização do autoconceito académico (Modelo MarshShavelson aninhado/bi-fatorial), o qual procura conciliar a existência de um autoconceito académico global com autoconceitos específicos nas diferentes matérias escolares. De acordo com este modelo, o autoconceito académico global afetaria diretamente as diferentes medidas específicas de autoconceito e não através das dimensões de primeira ordem, como propõem os modelos hierárquicos. Por outro lado, considera igualmente dimensões de primeira ordem correspondendo aos autoconceitos académicos específicos (e. g. autoconceito académico matemático, autoconceito académico verbal/língua materna). Deste modo, seria possível ter em consideração $\mathrm{o}$ caráter multifacetado do autoconceito académico e uma medida global deste constructo. Brunner e colaboradores (Brunner et al., 2009) testaram este modelo com os participantes do Programme for International Student Assessment (PISA), envolvendo estudantes de 26 países diferentes, e com estudantes luxemburgueses do $8^{\circ}$ ano (Brunner et al., 2010) revelando-se como um modelo mais ajustado aos dados do que os clássicos modelos hierárquicos. Assim, o modelo Marsh-Shavelson aninhado/bi-fatorial surge como uma alternativa aos modelos hierárquicos na explicação da organização estrutural do autoconceito académico.

Tendo presente que a organização do autoconceito varia com a idade (e.g., Byrne, 1996; Harter, 2012; Hattie, 1992; Shavelson et al., 1976), este estudo tem como principal objetivo adaptar a Escala de Autoconceito e Autoestima para Adolescentes (Peixoto, 2003; Peixoto \& Almeida, 1999) para a faixa etária dos préadolescentes. Deste modo, pretende-se analisar as propriedades psicométricas deste instrumento bem como testar a estrutura organizativa do autoconceito nesta faixa etária.

\section{Método}

\section{Participantes}

Participaram no estudo 774 estudantes de 12 escolas públicas da Região de Lisboa, dos quais 374 frequentavam o $5^{\circ}$ ano e 400 o $6^{\circ}$ ano de escolaridade, com a idade a variar entre os 9 e os 14 anos. Dos participantes, $50.1 \%$ eram raparigas, havendo $1.7 \%$ que não prestaram informação relativamente ao género. Relativamente ao sucesso escolar, 119 tiveram pelo menos uma situação de repetência no seu passado escolar.

\section{Instrumento}

A Escala de Autoconceito e Autoestima para Pré-adolescentes (EAAPA) foi construída a partir da Escala de Autoconceito e Autoestima para Adolescentes (Peixoto, 2003; Peixoto \& Almeida, 1999) a qual, por sua vez teve como ponto de partida a adaptação portuguesa do Self-Perception Profile for Adolescents (Peixoto, Alves-Martins, Mata, \& Monteiro, 1996, 1997). Relativamente ao Self-Perception Profile for Adolescents, a Escala de Autoconceito e Autoestima apresentava como principais diferenças o formato dos itens (apenas uma afirmação contra as duas da escala proposta 
por Harter, 1985), a adição de duas novas dimensões (Competência a Língua Materna e Competência a Matemática) e a estrutura proposta, uma vez que a nova escala pressupunha uma organização hierárquica do autoconceito (Peixoto, 2003; Peixoto \& Almeida, 2011). A presente escala radica nos mesmos pressupostos da escala para os adolescentes, diferindo desta pela exclusão de duas dimensões que parecem fazer menos sentido nesta fase do desenvolvimento: Atração Romântica e Amizades Íntimas. Assim, a primeira versão da EAAPA foi constituída por 43 itens distribuídos por 8 dimensões: Competência Escolar (5 itens - 1, 10, 19, 27 e 34), que pretende avaliar, de forma global, a perceção de competência nas tarefas escolares (e.g. "Alguns jovens percebem tudo o que os professores ensinam nas aulas"), Aceitação Social (5 itens - 2, 11, 20, 28 e 35), que inclui itens que reenviam para a perceção de integração social avaliada através da aceitação que sentem junto dos pares escolares (e.g. "Alguns jovens têm dificuldade em que os outros gostem deles"), Competência Atlética (5 itens - 3, 12, 21, 29 e 36) que reúne itens que pretendem avaliar a perceção do sujeito relativamente às suas capacidades físicas/atléticas (e.g. "Alguns jovens acham que são melhores a praticar desporto do que os outros jovens da sua idade"), Aparência Física (5 itens 4, 13, 22, 37 e 42) dimensão que pretende avaliar o grau de satisfação com o aspeto físico, peso, estatura, etc. (e.g. "Alguns jovens gostariam que o seu corpo fosse diferente"), Competência a Língua Materna (7 itens - 5,7,14, 16, 24, 31 e 40) que engloba itens que remetem para a perceção de competência verbal e escrita em Português (e.g. "Alguns jovens acham que escrevem bem"), Comportamento (5 itens $-6,15,23,30$ e 38 ) incluindo itens que pretendem avaliar a perceção de adequação do comportamento (e.g. "Alguns jovens arranjam complicações pela forma como se comportam"), Competência a Matemática (5 itens $-8,17,25,32$ e 41) uma dimensão que pretende avaliar a perceção de competência no domínio matemático (e.g. "Alguns jovens conseguem resolver problemas de Matemática muito rapidamente") e Autoestima (6 itens - 9, 18, 26, 33, 39 e 43) dimensão que pretende obter a avaliação global que a pessoa faz do seu valor enquanto pessoa, do grau de satisfação consigo própria, de forma global (e.g. "Alguns jovens gostam do tipo de pessoa que são"). Comparativamente à versão para adolescentes, nesta versão foram adicionados dois itens à dimensão Competência a Língua Materna devido à menor consistência interna desta dimensão na maioria dos estudos em que foi utilizada. Todos os itens são respondidos numa escala de 4 pontos variando entre "Exatamente como eu" e "Completamente diferente de mim". Cada item foi cotado de 1 a 4 (com a pontuação 1 significando baixa competência percebida e a pontuação 4 alta competência percebida), calculando-se, seguidamente, a média para cada dimensão da escala.

\section{Procedimentos de recolha e análise de dados}

Após a resposta positiva da Direção das escolas para a realização do estudo e da obtenção de autorização escrita da parte dos encarregados de educação, os alunos foram informados dos objetivos da pesquisa, sendo assegurada a sua participação voluntária e a confidencialidade dos dados recolhidos. A recolha dos protocolos procurou respeitar o princípio do anonimato, apesar de haver informações relativas à família e ao percurso escolar anterior do aluno. A avaliação ocorreu na sala de aula, tomando tempos cedidos pelos professores.

\section{Análise de dados}

Para testar a estrutura fatorial da escala de autoconceito e autoestima para pré-adolescentes utilizou-se a análise fatorial confirmatória. As análises foram realizadas utilizando o método de máxima verosimilhança disponível no AMOS 21.0 (Arbuckle, 2012). Para analisar o ajustamento global dos modelos utilizámos o quiquadrado, o Goodness of Fit Index (GFI), o índice de Tucker-Lewis (TLI), o Comparative Fit Index (CFI) e o Root Mean Square Error of Approximation (RMSEA). Uma vez que o teste do qui-quadrado é bastante sensível à dimensão da amostra (Bentler, 1990; Kline, 2011; Shumacker \& Lomax, 2004), e que o número de participantes envolvidos no presente estudo é relativamente elevado, baseámos as nossas decisões relativamente à adequação dos modelos nos 
restantes índices. Para o GFI, o CFI e o TLI podem considerar-se adequados valores superiores a 0.90 (Hair, William, Barry, Rolph, \& Ronald, 2010; Kline, 2011; Loehlin, 2004). Relativamente ao RMSEA, enquanto índice de desadequação aos dados, pretende-se que seja o menor possível. Deste modo, Browne e Cudeck (1993) sugerem que o valor de RMSEA deve situar-se abaixo de 0.08 e nunca ser superior a 0.10 ; por seu turno Steiger (1989), o criador do índice, refere que valores inferiores a 0.10 são bons e inferiores a 0.05 muito bons.

A fiabilidade foi analisada utilizando $\mathrm{o}$ coeficiente alfa de Cronbach e a fiabilidade compósita, para os quais se consideram adequados valores superiores a 0.70 (Hair et al., 2010). Os índices de fiabilidade compósita foram calculados utilizando a fórmula proposta por Geldhof, Preacher e Zyphur (2014). Aproveitando o facto de alguns participantes $(n=298)$ fazerem parte de um estudo longitudinal, calculámos a correlação teste-reteste, com um intervalo temporal de cerca de 12 meses, utilizando para o efeito o coeficiente de correlação de Pearson, com o objetivo de melhor conhecer a estabilidade temporal das diferentes dimensões.

$\mathrm{Na}$ comparação dos diferentes modelos em análise utilizámos a diferença nos valores do quiquadrado entre os modelos, o AIC (Akaike Information Criterion) e o BIC (Bayesian Information Criterion). Relativamente a estes, a preferência deverá ser por modelos que apresentem os valores mais baixos (Kline, 2011).

\section{Resultados}

Num primeiro momento testaram-se 2 modelos: um modelo unidimensional pressupondo um único fator e um modelo multidimensional pressupondo a existência de 7 fatores relacionados entre si (Figura 1 - Modelo Multidimensional). Não foram incluídos na análise os itens relativos à autoestima, uma vez que ao considerarmos este constructo como um julgamento global de valor, os itens que compõem essa escala poderiam contaminar os resultados das análises (e. g., Faria \& Fontaine, 1990). O modelo multidimensional apresentou índices de ajustamento global bastante superiores ao modelo unidimensional, no entanto com valores um pouco abaixo dos limites considerados aceitáveis (Quadro 1). A análise dos índices de modificação permitiu constatar a existência de cruzamentos de alguns itens com fatores diferentes daqueles em que seria suposto estarem incluídos. Assim sendo, optámos por diminuir os itens em cada fator retirando, em cada um, o item que apresentava valores de saturação mais baixos (no caso da perceção de competência a Língua Materna, retirámos os 3 itens com saturação mais baixa de modo a reter o mesmo número de itens das restantes dimensões específicas). Esta alteração possibilitou a obtenção de valores para os índices de ajustamento que podem ser considerados aceitáveis $\left(\chi^{2}(329)=1021.1 ; \quad \mathrm{GFI}=.90 ; \quad \mathrm{CFI}=.91 ; \quad \mathrm{TLI}=.90 ;\right.$ RMSEA $=.052[0.049,0.056])$, os quais são incrementados correlacionando alguns erros associados a itens dentro do mesmo fator (itens 6 e 23,23 e 30,30 e 38 todos da dimensão comportamento e os itens 2 e 11 da dimensão Aceitação Social). Estas alterações ao modelo permitiram um melhor ajustamento do modelo aos dados: $\quad \chi^{2}(325)=917.1 ; \quad$ GFI $=0.91 ; \quad$ CFI $=0.93$; TLI $=0.91 ;$ RMSEA $=0.049[0.045,0.052]$.

Quadro 1. Índices de ajustamento para os modelos unidimensional e multidimensional inicial

\begin{tabular}{lcccccc}
\hline \multicolumn{1}{c}{ Modelo } & $\chi^{2}$ & df & GFI & CFI & TLI RMSEA \\
\hline Nulo & 10707.4 & 595 & .37 & .00 & .00 & .148 \\
Uni & 6185.6 & 527 & .58 & .42 & .38 & .118 \\
dimensional & & & & & \\
Multi & 1677.8 & 539 & .87 & .89 & .88 & .052 \\
dimensional & &
\end{tabular}

Nota: GFI - Goodness-of-fit Index, CFI=Comparative fit Index; TLI=Tucker-Lewis Index, RMSEA=Root Mean Square Error of Approximation.

Relativamente à autoestima, testámos um modelo unidimensional considerando os 6 itens relativos a esta escala. Os valores obtidos, $\chi^{2}(9)=47.6 ; \quad$ GFI $=.98 ; \quad$ CFI $=.96 ; \quad$ TLI $=.94 ;$ RMSEA $=.074$ [0.054, 0.096], podem considerarse como indicando um bom ajustamento do modelo aos dados. $\mathrm{O}$ valor mais elevado para o 
Figura 1. Representação gráfica dos modelos testados. $\mathrm{CE}=$ Competência Escolar, $\mathrm{CM}=$ Competência Matemática, CLM=Competência a Língua Materna, $\mathrm{C}=$ Comportamento, AS=Aceitação Social, $\mathrm{CA}=$ Competência Atlética, $\mathrm{AF}=$ Aparência Física

Modelo Multidimensional

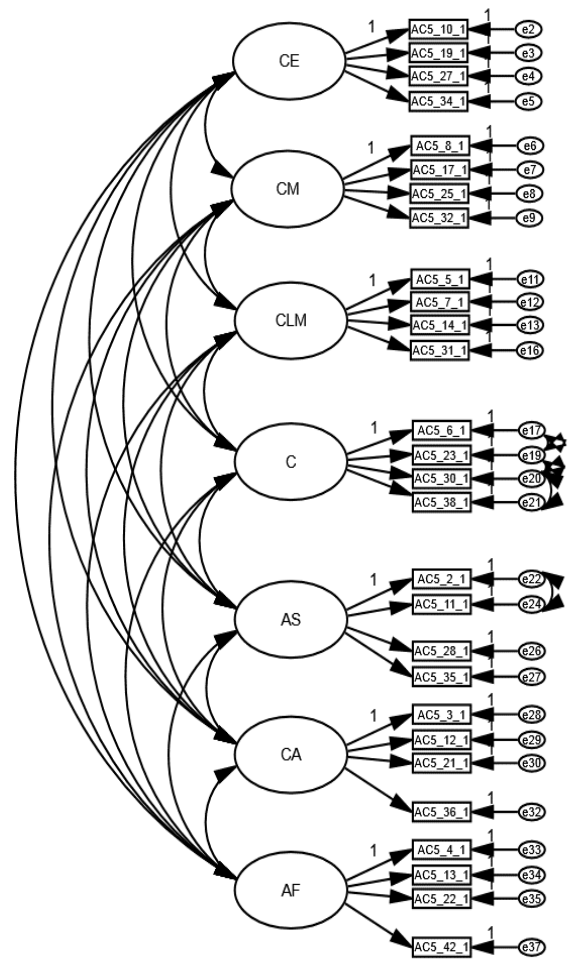

Modelo Hierárquico 2

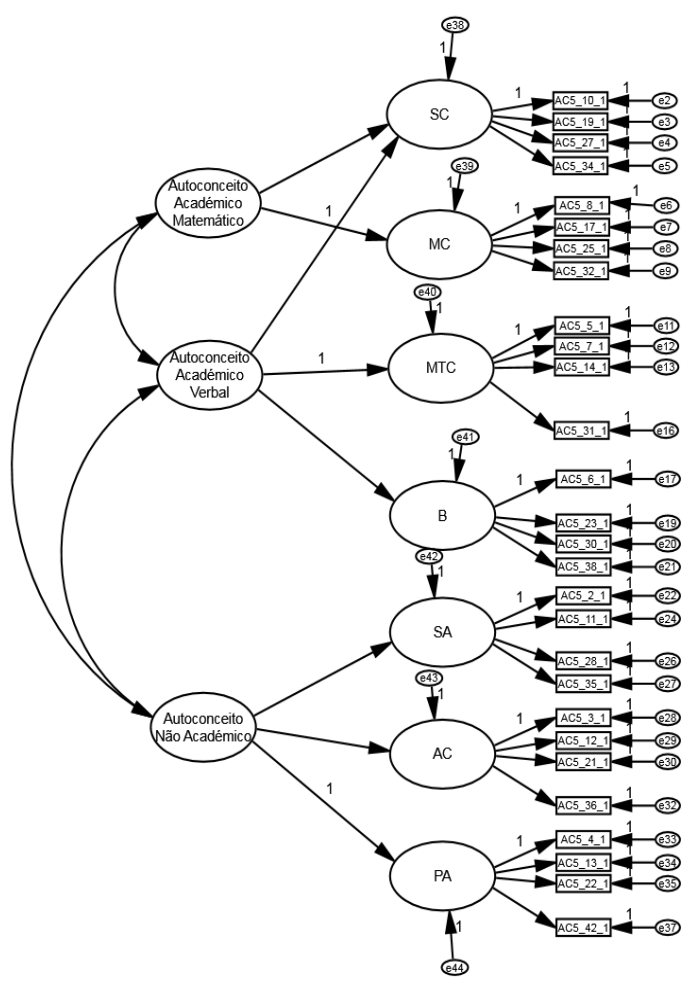

Modelo Hierárquico 1

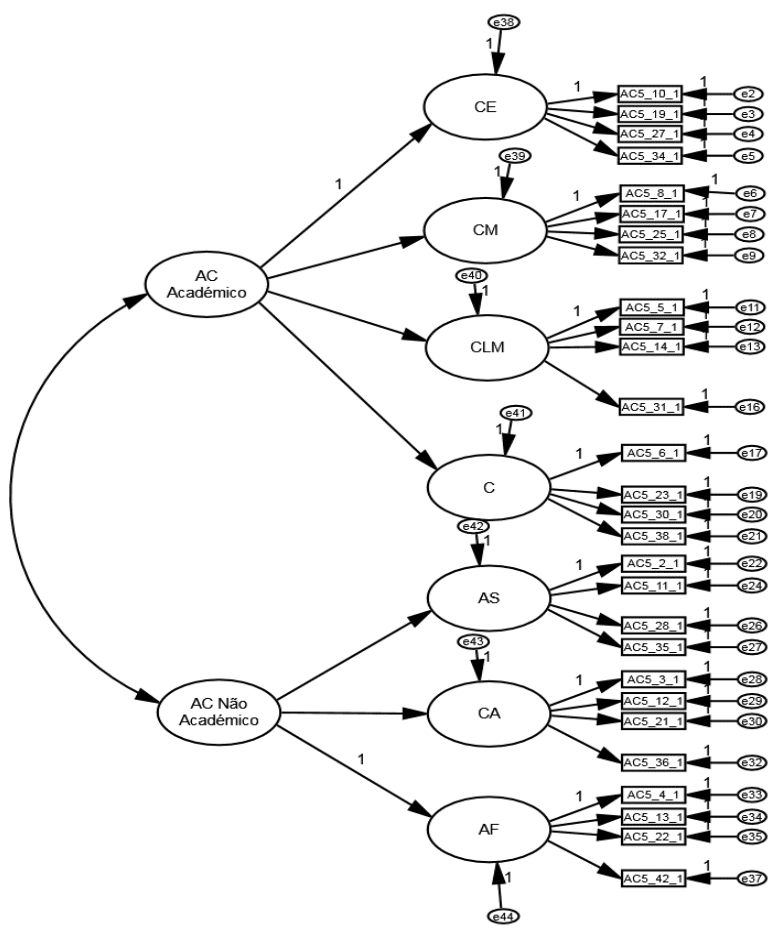

Modelo Bi-Fatorial aninhado 2 Fatores

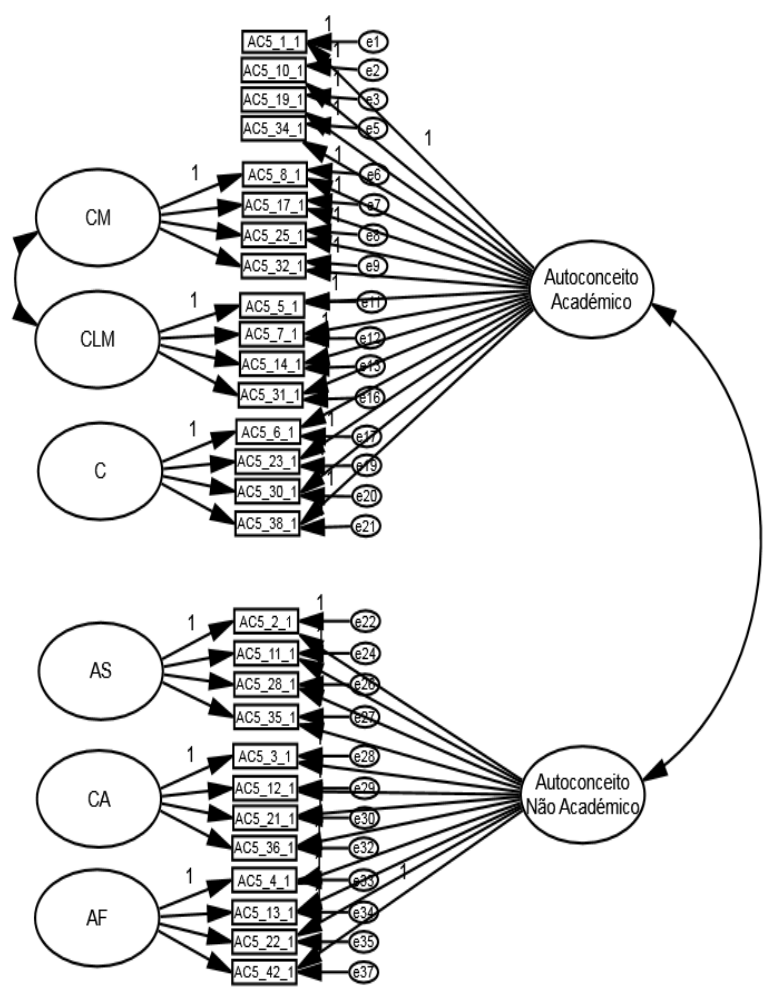


Quadro 2. Índices de ajustamento para os vários modelos testados

\begin{tabular}{lccccccc}
\hline \multicolumn{1}{c}{ Modelo } & $\chi^{2}$ & $d f$ & CFI & TLI & $\begin{array}{c}\text { RMSEA } \\
(\text { IC90\%) }\end{array}$ & AIC & BIC \\
\hline Nulo & 8306.9 & 378 & .00 & .00 & $\begin{array}{c}.165 \\
(.162, .169)\end{array}$ & 8362.9 & 8493.1 \\
$\begin{array}{l}\text { Multidimensional } \\
\text { ortogonal }\end{array}$ & 1963.3 & 348 & .80 & .78 & $\begin{array}{c}.077 \\
(.074, .081)\end{array}$ & 2079.3 & 2349.1 \\
$\begin{array}{l}\text { Multidimensional } \\
\text { correlacionado }\end{array}$ & 917.1 & 325 & .93 & .91 & $\begin{array}{c}.049 \\
(.045, .052)\end{array}$ & 1079.1 & 1455.9 \\
Hierárquico 1 & 1045.1 & 339 & .91 & .90 & $\begin{array}{c}.052 \\
(.048, .056)\end{array}$ & 1179 & 1490.7 \\
Hierárquico 2 & 977.9 & 338 & .92 & .91 & $\begin{array}{c}.049 \\
(.046, .053)\end{array}$ & 1113.9 & 1430.2 \\
$\begin{array}{l}\text { Bi-Fatorial } \\
\text { aninhado 2 }\end{array}$ & 901.6 & 322 & .93 & .91 & $\begin{array}{c}.048 \\
(.045, .052)\end{array}$ & 1069.6 & 1460.3 \\
\begin{tabular}{l} 
Fatores \\
\hline
\end{tabular} & & & & & & & \\
\hline
\end{tabular}

Nota: CFI=Comparative Fit Index; TLI=Tucker-Lewis Index, RMSEA=Root Mean Square Error of Approximation, $\mathrm{IC}=$ Intervalo de confiança, $\mathrm{AIC}=$ Akaike Information Criterion, $\mathrm{BIC}=$ Bayesian Information Criterion.

RMSEA é justificado pelo diminuto número de graus de liberdade, uma vez que este índice é penalizado em modelos com baixo número de variáveis e consequentes graus de liberdade (Kenny, Kaniskan, \& McCoach, 2014; Kenny \& McCoach, 2003). Por seu turno, nos indicadores mais estáveis como o CFI e o TLI (Kenny \& McCoach, 2003), os valores apresentados revelam um muito bom ajustamento do modelo.

Após a verificação da adequação do modelo multidimensional aos dados relativos ao autoconceito, testámos a sua organização. Tendo por base a investigação acerca da organização do autoconceito, a qual tem enfatizado a sua organização hierárquica (Abu-Hilal, 2000; Cheng \& Watkins, 2000; Marsh, 1990; Marsh \& Ayotte, 2003; Marsh \& Hocevar, 1985; Marsh \& Shavelson, 1985; Peixoto \& Almeida, 2011; Shavelson et al., 1976), começámos por testar modelos, organizando as diferentes dimensões hierarquicamente. A construção dos modelos hierárquicos sustentou-se na investigação com a Escala de Autoconceito e Autoestima para Adolescentes (Peixoto, 2003; Peixoto \& Almeida, 1999, 2011), assim como da investigação acerca da organização hierárquica do autoconceito atrás referida. De acordo com os estudos utilizando a versão desta escala com adolescentes (e.g., Peixoto, 2003; Peixoto \& Almeida, 1999, 2011), poderíamos supor a existência de 3 fatores de ordem superior (Autoconceito Académico, Autoconceito Social e Autoconceito de Apresentação). No entanto, a eliminação de duas dimensões nesta versão da escala, iria implicar que uma das dimensões de ordem superior (Autoconceito Social) fosse constituída por apenas um fator, uma vez que, para além da eliminação da dimensão Amizades Íntimas, estudos prévios com a escala dos adolescentes evidenciaram uma ligação mais forte da dimensão Comportamento com o Autoconceito Académico nos alunos mais novos. Assim, um dos modelos hierárquicos testados (Modelo Hierárquico 1) pressupõe a existência de dois fatores de ordem superior, um (Autoconceito Académico) incluindo as dimensões relacionadas com a escola (Competência Escolar, Competência a Língua Materna, Competência a Matemática e Comportamento) e o outro (Autoconceito não Académico) incluindo as restantes dimensões (Aceitação Social, Aparência Física e Competência Atlética). Um segundo modelo testado (Modelo Hierárquico 2) deriva do anterior, considerando a separação entre o autoconceito académico Matemático e o autoconceito académico Verbal, na linha da investigação realizada acerca da estrutura hierárquica do autoconceito académico (Marsh, 1990; Marsh \& Shavelson, 1985; Peixoto \& Almeida, 2011) e suportada no modelo do quadro de referência 
Figura 2. Coeficientes de regressão para o Modelo Bi-Fatorial aninhado 2 fatores

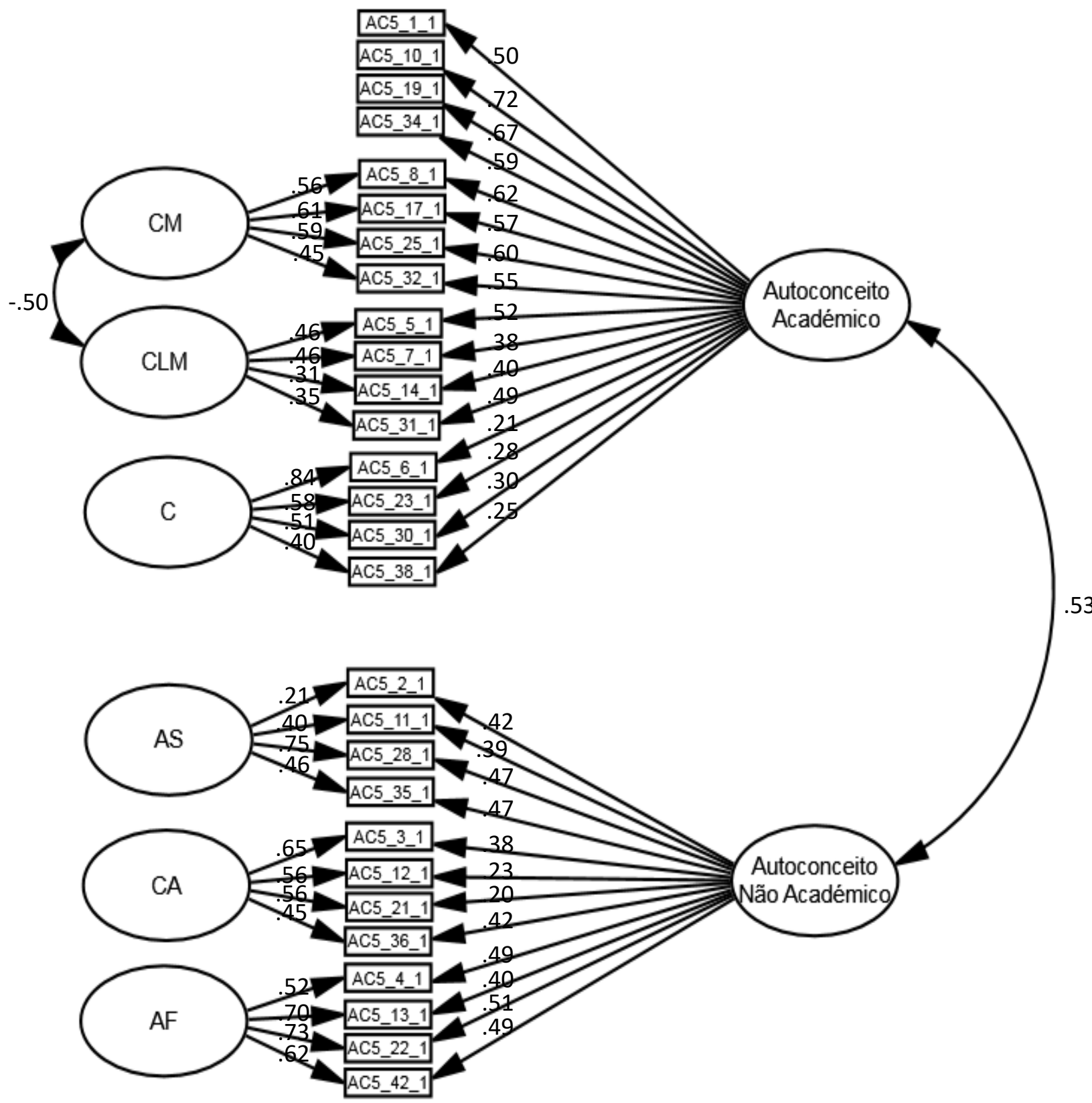

interno/externo (Marsh \& Hau, 2004; Möller, Pohlmann, Köller, \& Marsh, 2009). Por último, testámos um modelo bi-fatorial com dois fatores globais (Figura 1 - Modelo Bi-Fatorial aninhado 2 Fatores), inspirado nas investigações de Brunner e colaboradores sobre a organização do autoconceito académico (Brunner et al., 2010; Brunner et al., 2009) e dos trabalhos utilizando modelos bi-fatoriais (Chen, Hayes, Carver, Laurenceau, \& Zhang, 2012; Reise, 2012; Reise, Moore, \& Haviland, 2010). Dos quatro modelos testados (Figura 1), o Modelo Bi-fatorial aninhado
2 Fatores revela índices de ajustamento superiores aos restantes, com exceção do BIC que apresenta um valor ligeiramente superior ao do modelo hierárquico 2 (Quadro 2). Este resultado é compreensível se tivermos em consideração que o BIC favorece os modelos mais parcimoniosos (Garson, 2013; McCoach \& Black, 2008). Igualmente, a diferença nos valores do quiquadrado revela-se significativa na comparação com todos os modelos $\left(\Delta \chi^{2}(17)=143.5, p<.001\right.$, para o modelo hierárquico 1 e $\Delta \chi^{2}(16)=76.3$, $p<.001$, para o modelo hierárquico 2). $\mathrm{O}$ ajustamento do modelo hierárquico 2 também se 
Quadro 3. Estatísticas descritivas e fiabilidade para cada dimensão do autoconceito e para a autoestima

\begin{tabular}{|c|c|c|c|c|c|c|c|c|c|c|c|}
\hline & $M$ & $D P$ & $M d n$ & Assim. & Curt. & Mín. & Máx. & $\begin{array}{c}M \\
r_{\mathrm{i}(\mathrm{t}-\mathrm{i})}\end{array}$ & $\alpha$ & FC & CTR \\
\hline AA & 2.80 & .460 & 2.75 & .082 & .082 & 1 & 4 & .46 & .85 & .83 & .66 \\
\hline ANA & 2.92 & .489 & 2.92 & -.304 & .347 & 1 & 4 & .44 & .80 & .69 & .57 \\
\hline $\mathrm{CM}$ & 2.44 & .806 & 2.50 & .237 & -.666 & 1 & 4 & .74 & .88 & .88 & .73 \\
\hline CLM & 2.94 & .605 & 3.00 & -.432 & .020 & 1 & 4 & .47 & .69 & .69 & .48 \\
\hline $\mathrm{C}$ & 3.09 & .599 & 3.00 & -.397 & -.167 & 1 & 4 & .56 & .76 & .75 & .59 \\
\hline AS & 3.20 & .556 & 3.25 & -.876 & 1.35 & 1 & 4 & .53 & .73 & .73 & .39 \\
\hline $\mathrm{CA}$ & 2.60 & .670 & 2.50 & .007 & -.180 & 1 & 4 & .51 & .72 & .73 & .56 \\
\hline $\mathrm{AF}$ & 2.98 & .817 & 3.00 & -.654 & -.293 & 1 & 4 & .73 & .87 & .88 & .49 \\
\hline $\mathrm{AE}$ & 3.00 & .593 & 3.00 & -.586 & .597 & 1 & 4 & .54 & .78 & .79 & .54 \\
\hline
\end{tabular}

Nota: AA=Autoconceito Académico; ANA=Autoconceito Não-académico; $\mathrm{CM}=$ Competência Matemática, CLM=Competência a Língua Materna, $\mathrm{C}=$ Comportamento, AS=Aceitação Social, A=Competência Atlética, $\mathrm{AF}=$ Aparência Física.

diferencia significativamente do ajustamento do modelo hierárquico $1\left(\Delta \chi^{2}(1)=67.2, p<.001\right)$.

Os coeficientes de regressão para o modelo bi-fatorial aninhado 2 fatores apresentam-se na Figura 2. De uma forma geral, os coeficientes apresentam valores moderados a satisfatórios, variando entre -0.50 e 0.84 . O padrão observado é idêntico na maioria das dimensões, onde se registam coeficientes semelhantes tanto na própria dimensão como no fator associado, sendo em alguns casos ligeiramente superiores na dimensão específica. Observam-se como exceção os itens 8 e 32 da dimensão Competência a Matemática, 5 e 31 da dimensão Competência a Língua Materna e o item 2 da dimensão Aceitação social, cuja saturação é superior no fator não específico. Os itens não agrupados em qualquer dimensão apresentam coeficientes de regressão bastante satisfatórios no fator académico.

O Quadro 3 apresenta as estatísticas descritivas, a correlação item-total corrigida e as medidas de fiabilidade. A análise dos valores da assimetria e curtose sugerem uma distribuição Normal para as dimensões do autoconceito e para a autoestima, uma vez que, exceção feita à dimensão Aceitação Social, as restantes apresentam valores para a assimetria e curtose inferiores a |1|. A distribuição Normal das diferentes dimensões da escala de autoconceito e autoestima é ainda reforçada pelos valores próximos da média e mediana de cada fator. Por outro lado, os valores mínimo e máximo coincidem com os pontos extremos da escala de medida, refletindo uma boa dispersão dos resultados e suportando o poder discriminativo da escala.

Os valores para a fiabilidade podem considerar-se aceitáveis, com todas as dimensões a apresentarem valores superiores ao limiar comummente aceite (0.70), com exceção da dimensão de avaliação da auto-perceção de competência a língua materna, mas cujo coeficiente, tanto para o alfa de Cronbach, como para a fiabilidade compósita, se aproxima muito daquele valor. Para a análise da correlação testereteste há que ter em consideração investigações relativas a constructos idênticos que tenham aplicado um intervalo de tempo similar entre avaliações. Deste modo, os resultados obtidos para as dimensões Autoconceito Académico, Competência a Matemática, Competência a Língua Materna, Aceitação Social e Autoestima situam-se dentro dos valores encontrados em estudos similares (Chen, Yeh, Hwang, \& Lin, 2013; Gniewosz, Eccles, \& Noack, 2012; Green, Liem, Martin, Colmar, Marsh, \& McInerney, 2012; Guay, Ratelle, Roy, \& Litalien, 2010; Lindwall, Asci, \& Crocker, 2014; Morin, Maiano, Marsh, Janosz, \& 
Quadro 4. Matriz de intercorrelação entre as dimensões da EAAPA

\begin{tabular}{lcccccccc}
\hline \multicolumn{1}{c}{ Dimensão } & AA & ANA & CM & CLM & C & AS & CA & AF \\
\hline AA & - & & & & & & & \\
ANA & .33 & - & & & & & & \\
CM & .76 & .25 & - & & & & & \\
CLM & .68 & .24 & .25 & - & & & & \\
C & .57 & .13 & .15 & .27 & - & & & \\
AS & .30 & .68 & .15 & .26 & .20 & - & & \\
CA & .14 & .69 & .21 & $.05^{\mathrm{b}}$ & $-.07^{\mathrm{b}}$ & .29 & - & \\
AF & .28 & .77 & .18 & .21 & .15 & .30 & .22 & - \\
\hline
\end{tabular}

Nota: $\mathrm{AA}=$ Autoconceito Académico; $\mathrm{ANA}=$ Autoconceito Não-académico;

$\mathrm{CM}=$ Competência Matemática, CLM=Competência a Língua Materna,

$\mathrm{C}=$ Comportamento, $\mathrm{AS}=$ Aceitação Social, $\mathrm{CA}=$ Competência Atlética, $\mathrm{AF}=$ Aparência

Física. Todas as correlações são estatisticamente significativas $(p<.01)$, com exceção de ${ }^{\mathrm{b}}$.

Nagengast, 2011; Pinxten, Marsh, De Fraine, Van

Den Noortgate, \& Van Damme, 2014; Preckel, Niepel, Schneider, \& Brunner, 2013; Vogl \& Preckel, 2014; Wouters, De Fraine, Colpin, Van Damme, \& Verschueren, 2012) com idênticos constructos: correlações entre 0.55 e 0.62 para o Autoconceito Académico, entre 0.53 e 0.76 para o Autoconceito Matemático; entre 0.44 e 0.58 para o Autoconceito associado à Língua Materna; entre 0.30 e 0.47 para o Autoconceito Social e entre 0.48 e 0.58 para a Autoestima. Para as dimensões Competência Atlética e Aparência Física, os resultados situam-se um pouco abaixo dos valores encontrados em estudos análogos (Lindwall et al., 2014; Morin et al., 2011; Marsh et al., 2007; Preckel et al., 2013): correlações entre 0.69 e 0.74 para o Autoconceito Desportivo e entre 0.57 e 0.72 para a Imagem Corporal.

As correlações entre as dimensões da escala (Quadro 4) apresentam valores baixos a moderados, variando entre -0.07 e 0.33 , com excepção para as correlações entre as dimensões e os fatores a que estão associadas, isto é, entre a Competência a Matemática, Competência a Língua Materna e Comportamento e o Autoconceito Académico (0.76, 0.68 e 0.57 respectivamente), e entre a Aceitação Social, a Aparência Física e a Competência Atlética e o Autoconceito não Académico (0.68, 0.69 e 0.77, respectivamente). Por outro lado, observam-se correlações baixas a moderadas entre as dimensões específicas e os fatores não correspondentes, verificando-se uma clara diferenciação das dimensões específicas e dos fatores académico e não académico.

\section{Discussão}

Um dos objetivos deste trabalho foi adaptar a Escala de Autoconceito e Autoestima para Adolescentes (Peixoto, 2003; Peixoto \& Almeida, 1999) para a faixa etária dos pré-adolescentes. Os resultados obtidos revelaram que é possível discriminar, neste nível etário, sete dimensões do autoconceito relacionadas entre si. Estas dimensões demonstraram, igualmente, um bom poder discriminativo, ao apresentarem uma distribuição Normal e como valores mínimos e máximos os extremos da escala de medida. Relativamente à fiabilidade, as diferentes dimensões apresentaram valores adequados, quer para o alfa de Cronbach quer para a fiabilidade compósita. A estabilidade temporal das diferentes dimensões do autoconceito revelou-se igualmente adequada, tendo em conta o intervalo temporal considerado e os estudos de referência (Chen et al., 2013; Gniewosz et al., 2012; Green et al., 2012; Guay et al., 2010; Lindwall et al., 2014; Morin et al., 2011; Marsh et al., 2007; Pinxten et al., 2014; Preckel et al., 2013; Vogl \& Preckel, 2014; Wouters et al., 2012).

$\mathrm{O}$ modelo unidimensional testado para a dimensão da autoestima mostrou-se bem ajustado aos dados, apresentando-se esta medida igualmente adequada tanto relativamente à fiabilidade como à estabilidade temporal.

Em suma, podemos afirmar que a EAAPA é um instrumento de avaliação das representações de si, que apresenta validade interna e bons indicadores métricos, nomeadamente no que respeita à fiabilidade e à estabilidade temporal, na 
utilização com pré-adolescentes. Comparativamente ao SPPC de Harter (1982), a presente escala tem a vantagem de permitir uma avaliação mais precisa e diferenciada do autoconceito académico, uma vez que considera diferentes vertentes específicas deste autoconceito (língua materna e matemática) para além de uma avaliação global (Harter 1982, 1985; Alves-Martins, Peixoto, Mata, \& Monteiro, 1995). Por sua vez, relativamente ao SDQ de Marsh (Faria \& Fontaine, 1990; Marsh, et al., 1984) apresenta menos itens por dimensão, o que constitui uma vantagem quando se procura uma prova de aplicação mais rápida.

Relativamente à organização estrutural do autoconceito os resultados confirmam a sua estrutura multidimensional, corroborando os resultados obtidos em diversos estudos anteriores que avaliaram a estrutura multidimensional do autoconceito (e.g., Cheng \& Watkins, 2000; Hattie, 1992; Marsh \& Shavelson, 1985; Marsh, 1990; Molina et al., 2011; Shavelson \& Bolus, 1982). Os resultados demonstram, assim, que os pré-adolescentes são capazes de avaliar a sua competência atendendo às diferentes dimensões da sua vida, sendo adequado considerar uma estrutura multidimensional do autoconceito e cada vez mais diferenciada nestas faixas etárias (Byrne, 1996; Faria \& Fontaine, 1990; Harter, 2012; Hattie, 1992; Marsh et al., 1984, Molina et al., 2011).

Apesar de os modelos que contemplavam uma estrutura hierárquica do autoconceito terem demonstrado bons índices de ajustamento, quando analisados individualmente, foi possível observar que o modelo Marsh-Shavelson aninhado/bifactorial foi o que apresentou a melhor adequação aos dados. Estes resultados vêm corroborar a hipótese, já levantada na literatura, de que a organização do autoconceito, embora hierárquica, será bem mais complexa do que a proposta pelo modelo de Marsh/Shavelson (1985).Os modelos de Shavelson e colaboradores (1976) e o modelo de Marsh/Shavelson (Marsh, 1990; Marsh \& Hocevar, 1985; Marsh \& Shavelson, 1985; Marsh \& Ayotte, 2003) surgem como os mais inspiradores e os mais extensivamente estudados, no que concerne à avaliação da estrutura do autoconceito. Os autores consideram a existência de dois fatores académicos de ordem superior -
Matemática e Verbal - em vez de um único fator académico de ordem superior. Mais recentemente, e apoiando a ideia de que a estrutura do autoconceito é menos clara do que a inicialmente proposta por estes modelos, principalmente no que se refere ao autoconceito académico, as investigações de Brunner e colaboradores (2010; Brunner et al., 2009) vieram propor um outro modelo explicativo para a organização do autoconceito, mais especificamente do autoconceito académico - o modelo bi-fatorial.

$\mathrm{O}$ modelo com melhor ajustamento encontrado neste estudo corrobora os resultados encontrados por Brunner e colaboradores (2010; Brunner et al., 2009) relativamente ao autoconceito académico. Neste contexto, a utilização de modelos bi-fatoriais surge como uma tendência explicativa da organização de constructos psicológicos, como é o caso da perceção de competência, ao permitir dar conta, em simultâneo, tanto da existência de constructos gerais como de facetas relacionadas com o constructo global (Chen et al., 2012; Reise, 2012; Reise et al., 2010). Este modelo oferece algumas vantagens em relação aos modelos hierárquicos clássicos, na medida em que contempla tanto os constructos mais gerais como as dimensões mais específicas, separando a variância comum partilhada por todos os itens da variância associada a cada fator (Chen et al., 2012; Reise et al., 2010). Por este motivo, é possível compreender em que medida os itens refletem o constructo geral sem ter impacto na sua multidimensionalidade e compreender também a forma como estes refletem constructos específicos (Reise et al., 2010). Se o intuito for avaliar constructos latentes (e.g. o autoconceito académico e o autoconceito não académico) e as suas dimensões específicas (e.g, competência matemática), como dimensões de primeira ordem, a variância latente reflete tanto a variância dos domínios específicos do autoconceito contemplados no modelo bi-fatorial (e.g., competência a matemática) como a variância dos domínios mais gerais (e.g., autoconceito académico) (Brunner et al., 2009).

A confirmação deste modelo permite ter em conta vários aspetos teóricos considerados noutros modelos do autoconceito, nomeadamente: a sua 
natureza multidimensional (representada neste estudo por sete dimensões); a sua organização hierárquica (ao considerar as dimensões de primeira ordem e as dimensões de ordem superior); a separação entre os domínios verbal e matemático proposta no modelo de Marsh/Shavelson; e a existência de dois autoconceitos gerais no topo da hierarquia (autoconceito académico e autoconceito não académico), como contemplado no modelo de Shavelson e colaboradores. Considerando-se a estrutura bi-fatorial do autoconceito, será possível obter-se um modelo integrativo de outros modelos teóricos consolidados na literatura e que, à partida, seriam considerados incompatíveis (Brunner et al., 2010; Brunner et al., 2009).

Em suma, os resultados encontrados neste estudo refletem, por um lado, a natureza complexa da estrutura do autoconceito e, por outro, apoiam um modelo recente na literatura que permite integrar aspetos distintos, e aparentemente não conciliáveis, da estrutura organizativa do autoconceito. Por fim, este estudo contribui para uma maior compreensão da organização do autoconceito em faixas etárias mais precoces, os pré-adolescentes.

O modelo bi-fatorial poderá ter algumas implicações práticas interessantes, nomeadamente no que diz respeito à intervenção educativa na área do autoconceito. Se, por um lado, a sua estrutura multidimensional permite que sejam considerados e trabalhados autoconceitos específicos, consoante as necessidades dos sujeitos, por outro lado, atendendo à sua relação e organização em autoconceitos mais gerais, poderse-á verificar o impacto destas intervenções nas dimensões mais globais do autoconceito (Brunner et al., 2009).

Apesar do contributo do presente trabalho para o estudo da organização do autoconceito, outros aspetos importantes deverão ser considerados em futuras investigações. Deste modo, parece-nos pertinente verificar se, por um lado, tal como sucedia relativamente aos modelos clássicos de organização do autoconceito, também com os modelos bi-fatoriais se confirma a invariância estrutural relativamente a variáveis como o género, a idade e o estatuto escolar. Por outro lado, importa igualmente perceber, até que ponto este modelo de organização aplicado à estrutura do autoconceito académico poderá ser extensível a outras áreas do autoconceito como sejam o físico ou o artístico. Em suma, os nossos resultados são interessantes e entusiasmantes, particularmente no que se refere à análise hierárquica do autoconceito em pré-adolescentes. $\mathrm{O}$ instrumento aqui desenvolvido certamente necessita de mais investigações com o objetivo de replicar os nossos resultados e, talvez, explorar a pertinência de incluir outras dimensões no autoconceito académico.

\section{Referências}

Abu-Hilal, M. M., \& Bahri, T. M. (2000). Selfconcept: The generalizability of research on the SDQ, Marsh/Shavelson model and $\mathrm{I} / \mathrm{E}$ frame of reference model to United Arab Emirates students. Social Behavior and Personality, 28, 309-322. doi:10.2224/sbp. 20 00.28.4.309

Alves Martins, M., Peixoto, F., Mata, L., \& Monteiro, V. (1995). Escala de autoconceito para crianças e pré-adolescentes de Susan Harter. In L. S. Almeida, M. R. Simões, \& M. M. Gonçalves (Eds.), Provas psicológicas em Portugal (pp. 79-89). Braga: APPORT.

Arbuckle, J. L. (2012). Amos (Version 21.0) [Computer Program]. Chicago: SPSS.

Barros, A. F. (2012). Características psicométricas da adaptação portuguesa do perfil de auto percepção para estudantes universitários SPPCS. Revista Iberoamericana de Diagnóstico y Evaluación - e Avaliação Psicológica, 33, 93-110.

Bentler, P. M. (1990). Comparative fit indexes in structural models. Psychological Bulletin, 107, 238-246. doi:10.1037/00332909.107.2.238

Browne, M. W., \& Cudeck, R. (1993). Alternative ways of assessing model fit. In K. A. Bollen \& J. S. Long (Eds.), Testing structural equation models (pp. 136-162). Newbury Park, California: Sage.

Brunner, M., Keller, U., Dierendonck, C., Reichert, M., Ugen, S., Fischbach, A., \& Martin, R. (2010). The structure of academic self-concepts revisited: The nested 
Marsh/Shavelson model. Journal of Educational Psychology, 102, 964-981. doi:10.1037/a0019644

Brunner, M., Keller, U., Hornung, C., Reichert, M., \& Martin, R. (2009). The cross-cultural generalizability of a new structural model of academic self-concepts. Learning and Individual Differences, 19, 387-403. doi:10.1016/j.lindif.2008.11.008

Byrne, B. (1996). Measuring self-concept across life span: Issues and instrumentation. Washington: APA.

Byrne, B., \& Shavelson, R. (1996). On the structure of social self-concept for pre-, early, and late adolescentes: A test of the Shavelson, Hubner, and Stanton (1976) model. Journal of Personality and Social Psychology, 70, 599613. doi:10.1037/0022-3514.70.3.599

Chen, F., Hayes, A., Carver, C., Laurenceau, J. P., \& Zhang, Z. (2012). Modeling general and specific variance in multifaceted constructs: A comparison of the bifactor model to other approaches. Journal of Personality, 80, 219251. doi:10.1111/j.1467-6494.2011.00739.x

Chen, S., Yeh, Y., Hwang, F., \& Lin, S. J. (2013). The relationship between academic selfconcept and achievement: A multicohortmultioccasion study. Learning and Individual Differences, $\quad 23, \quad 172-178$. doi:10.1016/j.lindif.2012.07.021

Cheng, C. H. K, \& Watkins, D. (2000). Age and gender invariance of self-concept factor structure: An investigation of a newly developed Chinese self-concept instrument. International Journal of Psychology, 35, 186193. doi:10.1080/00207590050171120

Faria, L., \& Fontaine, A. M. (1990). Avaliação do conceito de si próprio de adolescentes: adaptação do SDQ I de Marsh à população portuguesa. Cadernos de Consulta Psicológica, 6, 97-105.

Garson, G.D. (2013). Hierarchical linear modeling: Guide and applications. Thousand Oaks: Sage Publications.

Geldhof, G., Preacher, K. J., \& Zyphur, M. J. (2014). Reliability estimation in a multilevel confirmatory factor analysis framework. Psychological Methods, 19, 72-91. doi:10.1037/a0032138
Gniewosz, B., Eccles, J. S., \& Noack, P. (2012). Secondary school transition and the use of different sources of information for the construction of the academic self-concept. Social Development, 21, 537-557. doi:10.1111/j.1467-9507.2011.00635.x

Gómez-Maqueo, E. L., Eguiarte, B. E. B, \& Patiño, C. D. (2007). Estructura factorial del inventario autodescriptivo del adolescente (IADA). Revista Iberoamericana de Diagnóstico y Evaluación - e Avaliação Psicológica, 23, 149-173.

Green, J., Liem, G. D., Martin, A. J., Colmar, S., Marsh, H. W., \& McInerney, D. (2012). Academic motivation, self-concept, engagement, and performance in high school: Key processes from a longitudinal perspective. Journal of Adolescence, 35, 1111-1122.

doi:10.1016/j.adolescence.2012.02.016

Guay, F., Ratelle, C. F., Roy, A., \& Litalien, D. (2010). Academic self-concept, autonomous academic motivation, and academic achievement: Mediating and additive effects. Learning and Individual Differences, 20, 644653. doi:10.1016/j.lindif.2010.08.001

Hair, J. F., William, C. B., Barry, J. B., Rolph, E. A., \& Ronald, L. T. (2010). Multivariate data analysis $\left(7^{\text {th }}\right.$ ed.). New Jersey: Pearson Education.

Harter, S. (1982). The perceived competence scale for children. Child Development, 53, 87-97. doi: $10.2307 / 1129640$

Harter, S. (1985). Manual for the self-perception profile for children. Denver: University of Denver.

Harter, S. (1988). Manual for the self-perception profile for adolescents. Denver: University of Denver.

Harter, S. (2012). The construction of the self: Developmental and sociocultural foundations $\left(2^{\text {nd }} E d.\right)$. New York: Guilford Press.

Hattie, J. (1992). Self-concept. Hillsdale: Lawrence Erlbaum.

Jackson, S. A., Thomas, P. R., \& Marsh, H. W. (2001). Relationships between flow, selfconcept, psychological skills, and performance. Journal of Applied Sport Psychology, 13, 129-153. doi:10.1080/104132 


\section{5}

Kenny, D. A., \& McCoach, D. B. (2003). Effect of the number of variables on measures of fit in structural equation modeling. Structural Equation Modeling, 10, 333-351. doi:10.1207/S15328007SEM1003_1

Kenny, D. A., Kaniskan, B., \& McCoach, D. B. (2014). The performance of RMSEA in models with small degrees of freedom. Sociological Methods \& Research. Advance online publication. doi:10.1177/0049 124114543236

Kline, R. B. (2011). Principles and practice of structural equation modeling ( $3^{\text {rd }}$ Ed.). New York: Guilford Press.

Leung, K. C., Marsh, H., Yeung, A. S., \& Abduljabbar, A. S. (2015). Validity of social, moral and emotional facets of self-description questionnaire. Journal of Experimental Education, 83, 1-23. doi:10.1080/00220973.2 013.876229

Lindwall, M., Asci, H., \& Crocker P. (2014).The physical self in motion: Within-person change and associations of change in self-esteem, physical self-concept, and physical activity in adolescent girls. Journal of Sport \& Exercise Psychology, 36, 551-563. doi:10.1123/jsep.20 13-0258

Loehlin, J. C. (2004). Latent variable models: An introduction to factor, path and structural analysis (4 ${ }^{\text {th }}$ Ed.). Mahwah, NJ: Lawrence Erlbaum.

Marsh, H. W. (1986). Verbal and math selfconcepts: An internal/external frame of reference model. American Educational Research Journal, 23, 129-149. doi:10.1080/104132001753149865

Marsh, H. W. (1990). The structure of academic self-concept: The Marsh/Shavelson model. Journal of Educational Psychology, 82, 623636. doi:10.1037/0022-0663.82.4.623

Marsh, H. W., \& Ayotte, V. (2003). Do multiple dimensions of self-concept become more differentiated with age? The differential distinctiveness hypothesis. Journal of Educational Psychology, 95, 687-706. doi:10.1037/0022-0663.95.4.687

Marsh, H. W., Barnes, J., Cairns, L., \& Tidman, M. (1984). Self-description questionnaire:
Age and sex effects in the structure and level of self-concept for preadolescent children. Journal of Educational Psychology, 76, 940956. doi:10.1037/0022-0663.76.5.940

Marsh, H. W., Craven, R. G., \& Martin, A. J. (2006). What is the nature of self-esteem? Unidimensional and multidimensional perspectives. In M. H. Kernis, \& M. H. Kernis (Eds.), Self-esteem issues and answers: A sourcebook of current perspectives (pp. 1624). New York, US: Psychology Press.

Marsh, H. W., Gerlach, E., Trautwein, U., Ludtke, O., \& Brettschneider, W. (2007). Longitudinal study of preadolescent sport self-concept and performance: Reciprocal effects and causal ordering. Child Development, 78, 1640-1656. doi:10.1111/j.1467-8624.2007.01094.x

Marsh, H. W., \& Hattie, J. (1996). Theoretical perspectives on the structure of self-concept. In B. A. Bracken (Ed.), Handbook of selfconcept: Developmental, social and clinical considerations (pp. 38-90). New York: John Wiley \& Sons.

Marsh, H. W., \& Hau, K. (2004). Explaining paradoxical relations between academic selfconcepts and achievements: Cross-cultural generalizability of the internal/external frame of reference predictions across 26 countries. Journal of Educational Psychology, 96, 5667. doi:10.1037/0022-0663.96.1.56

Marsh, H. W., \& Hocevar, D. (1985). Application of confirmatory factor analysis to the study of self-concept: First- and higher-order factor models and their invariance across groups. Psychological Bulletin, 97, 562-582. doi:10.1037/0033-2909.97.3.562

Marsh, H. W., \& Shavelson, R. (1985). Selfconcept: Its multifaceted, hierarchical structure. Educational Psychologist, 20, 107123. doi:10.1207/s15326985ep2003_1

Marsh, H. W., Hey, J., Johnson, S., \& Perry, C. (1997). Elite athlete Self-Description Questionnaire: Hierarchical confirmatory factor analysis of responses by two distinct groups of elite. International Journal of Sport Psychology, 28, 237-258.

McCoach, D. B., \& Black, A. C. (2008). Assessing model adequacy. In A. A. O'Connell, \& D. B. McCoach (Eds.), 
Multilevel modeling of educational data (pp. 245-272). Charlotte, NC: Information Age Publishing.

Molina, M. F., Raimundi, M. J., López, C., Cataldi, S., \& Bugallo, L. (2011). Adaptación del perfil de autopercepciones para niños para su uso en la ciudad de Buenos Aires. Revista Iberoamericana de Diagnóstico y Evaluación - e Avaliação Psicológica, 32, 53-78.

Möller, J., Pohlmann, B., Köller, O., \& Marsh, H. W. (2009). A meta-analytic path analysis of the internal/external frame of reference model of academic achievement and academic selfconcept. Review of Educational Research, 79, 1129-1167. doi:10.3102/0034654309337522

Morin, A. S., Maiano, C., Marsh, H. W., Janosz, M., \& Nagengast, B. (2011). The longitudinal interplay of adolescents' self-esteem and body image: A conditional autoregressive latent trajectory analysis. Multivariate Behavioral Research, 46, 157-201. doi:10.1080/00273171 .2010 .546731

Peixoto, F. (2003). Auto-estima, autoconceito e dinâmicas relacionais em contexto escolar. Tese de Doutoramento. Universidade do Minho.

Peixoto, F. (2011). "Is it beneficial to stress grades to my child?" - Relationships between parental attitudes towards academic achievement, motivation, academic selfconcept and academic achievement in adolescents. International Journal about Parents in Education, 5, 97-108.

Peixoto, F., \& Almeida, L. S. (1999). Escala de auto-conceito e auto-estima. In A. P. Soares, S. Araújo, \& S. Caires (Eds.), Avaliação psicológica: Formas e contextos (Vol. VI, pp. 632-640). Braga: APPORT.

Peixoto, F., \& Almeida, L. S. (2010). Selfconcept, self-esteem and academic achievement: Strategies for maintaining selfesteem in students experiencing academic failure. European Journal of Psychology of Education, 25, 157-175. doi:10.1007/s10212010-0011-z

Peixoto, F., \& Almeida, L. S. (2011). A organização do autoconceito: Análise da estrutura hierárquica em adolescentes.
Psicologia: Reflexão e Crítica, 24, 533-541. doi:10.1590/S0102-79722011000300014

Peixoto, F., Alves-Martins, M., Mata, L., \& Monteiro, V. (1996). Adaptação da escala de auto-conceito para adolescentes de Susan Harter para a população portuguesa. In L. S. Almeida, S. Araújo, M. M. Gonçalves, C. Machado, M. R. Simões (Eds.) Avaliação psicológica: Formas e contextos (Vol. IV, pp. 531-537). Braga: APPORT.

Peixoto, F., Alves-Martins, M., Mata, L., \& Monteiro, V. (1997). Escala de auto-conceito para adolescentes de Susan Harter. In Actas da $V$ conferência internacional: Avaliação psicológica: formas e contextos. Braga: APPORT.

Peixoto, F., \& Carvalho, R. (2009). Parental attitudes toward academic achievement: Effects on motivation, self-concept and school achievement. In M. Wosnitza, S. Karabenick, A. Efklides, \& P. Nenniger (Eds.), Contemporary motivation research: From global to local perspectives (pp. 279-297). Göttingen \& New York: Hogrefe \& Huber.

Pinxten, M., Marsh, H. W., De Fraine, B., Van Den Noortgate, W., \& Van Damme, J. (2014). Enjoying mathematics or feeling competent in mathematics? Reciprocal effects on mathematics achievement and perceived math effort expenditure. British Journal of Educational Psychology, 84, 152-174. doi:10.1111/bjep.12028

Preckel, F., Niepel, C., Schneider, M., \& Brunner, M. (2013). Self-concept in adolescence: A longitudinal study on reciprocal effects of self-perceptions in academic and social domains. Journal of Adolescence, 36, 11651175. doi:10.1016/j.adolescence.2013.09.001

Reise, S. (2012). The rediscovery of bifactor measurement models. Multivariate Behavioral Research, 47, 667-696. doi:10.1080/002731 71.2012 .715555

Reise, S., Moore, T., \& Haviland, M. (2010). Bifactor models and rotations: Exploring the extent to which multidimensional data yield univocal scales scores. Journal of Personality Assessment, 92, 544-559. doi:10.1080/002238 91.2010 .496477 
Shavelson, R. J., \& Bolus, R. (1982). Selfconcept: The interplay of theory and methods. Journal of Educational Psychology, 74, 3-17. doi:10.1037/00220663.74.1.3

Shavelson, R. J., Hubner, J. J., \& Stanton, G. C. (1976). Self-concept: Validation of construct interpretations. Review of Educational Research, 46, 407-441. doi:10.3102/0034654 3046003407

Shumacker, R. E., \& Lomax, R. G. (2004). A beginner's guide to structural equation modeling ( $2^{\text {nd }}$ Edition). Mahwah, $\mathrm{NJ}$ : Lawrence Erlbaum.

Steiger, J. H. (1989). Ezpath: Causal modeling. Evanston: Systat.

Vispoel, W. P. (1995). Self-concept in artistic domains: An extension of the Shavelson, Hubner, and Stanton (1976) model. Journal of Educational Psychology, 87, 134-153. doi:10.1037/0022-0663.87.1.134

Vogl, K., \& Preckel, F. (2014). Full-time ability grouping of gifted students: Impacts on social self-concept and school-related attitudes. Gifted Child Quarterly, 58, 51-68. doi:10.1177/0016986213513795

Wouters, S., De Fraine, B., Colpin, H., Van Damme, J., \& Verschueren, K. (2012). The effect of track changes on the development of academic self-concept in high school: A dynamic test of the big-fish-little-pond effect. Journal of Educational Psychology, 104, 793805. doi:10.1037/a0027732 